\title{
Atopic triad (allergic rhinitis, eczema, and asthma) and eye rubbing as risk factors for keratoconus: a systematic review and meta-analysis
}

\author{
Abdulmajeed Albalawi ${ }^{1 *}$, Alanoud Alharbi ${ }^{2}$, Hussain Alhasani ${ }^{3}$, Amal Alharbi ${ }^{1}$, \\ Raghad Abdullah ${ }^{4}$, Abrar Alamrani ${ }^{1}$, Amal Althobaiti ${ }^{1}$, Ziad Albalwi ${ }^{1}$, Yassmeen Alblowi ${ }^{1}$, \\ Nuwayr Albalawi ${ }^{1}$, Nujud Albalawi ${ }^{1}$, Atheer Alhwaiti ${ }^{1}$, Turki Fehaid Algethami ${ }^{5}$, \\ Dina Abusabir', Abeer Kalbouneh ${ }^{6}$
}

\author{
${ }^{1}$ Faculty of Medicine, Tabuk University, Tabuk, Saudi Arabia \\ ${ }^{2}$ Department of Family Medicine, King Fahad Specialist Hospital, Buraydah, Qassim, Saudi Arabia \\ ${ }^{3}$ College of Medicine, Umm Al Qura University, Al-Qunfudah, Saudi Arabia \\ ${ }^{4}$ The program of Medicine, Ibn Sina National College, Jeddah, Saudi Arabia \\ ${ }^{5}$ College of Medicine, Taibah University, Medina, Saudi Arabia \\ ${ }^{6}$ Faculty of Medicine, Gezira University, Sudan
}

Received: 15 March 2021

Revised: 31 March 2021

Accepted: 01 April 2021

\section{*Correspondence:}

Dr. Abdulmajeed Albalawi,

E-mail: abdulmajeed.a489@gmail.com

Copyright: ( $)$ the author(s), publisher and licensee Medip Academy. This is an open-access article distributed under the terms of the Creative Commons Attribution Non-Commercial License, which permits unrestricted non-commercial use, distribution, and reproduction in any medium, provided the original work is properly cited.

\begin{abstract}
Keratoconus $(\mathrm{KN})$, is an eye disorder, characterized by progressive thinning and protrusion of central cornea. A number of conditions such as such as allergy, asthma, eczema, and eye rubbing has been shown to be associated with the development of $\mathrm{KN}$. However, there is a disagreement regarding some of risk factors and their strength so we conducted systematic review and meta-analysis to determine how strongly risk factors such as eye rubbing, and atopic triad associate with $\mathrm{KN}$ development and progression. We systematically searched the literature for related studies using specific keywords and key phrases. The studies were scrutinized based on inclusion and exclusion criteria. Finally, we extracted relevant qualitative and quantitate information from studies. For meta-analysis we used odds ratio (OR) and their $95 \%$ CI were used to draw forest plots. 35 studies were selected in final meta-analysis. Our meta-analysis yielded the combined effect of risk factors with OR of 2.20 and a $95 \%$ CI of 1.84-2.64. Furthermore, we found that eye rubbingrelated studies had effect size of OR 2.09 with a $95 \%$ CI of $1.76-2.49$ and a p value of 0.00001 . For atopic triad (allergic rhinits, asthma and eczema) related studies, the meta-analysis yielded overall effect size of OR 2.34 with $95 \%$ CI of 2.06-2.66. Eye rubbing and atopic triad (allergic rhinitis, eczema, and asthma) are important risk factors for KC development with statistically strong association.
\end{abstract}

Keywords: Keratoconus, Eye rubbing, Allergic rhinitis, Eczema, Asthma, Atopy

\section{INTRODUCTION}

Keratoconus (KC), an eye disorder, characterized by thinning and outward bulging of cornea into a cone shape. ${ }^{1}$ If not treated in time, it can lead to blindness if irregular astigmatic continues to progress. ${ }^{2}$ According to the statistics, the prevalence of $\mathrm{KC}$ ranges from 1 in 500000 to 20 in 100000 in different populations. ${ }^{3}$ The prevalence rates and factors differ depending on the location and race. ${ }^{4}$ For example, Asian populations have higher prevalence compared to the White. ${ }^{5} \mathrm{KCwas}$ first reported in last half of the $19^{\text {th }}$ century by John Nottingham. ${ }^{6}$ 
There are a number of factors involved in development and progression of disease including genetic and environmental factors. At genetic level, more than one gene is involved and it tends to run in families. ${ }^{7}$ Environmental factors play crucial role in disease development even the individuals have genetic susceptibility. Epidemiological studies have shown that both genders are affected equally with men having slightly higher tendency. ${ }^{8}$ The symptoms can appear in any age but in most cases in second decade of life. The symptoms range from reduced fluctuating vision to astigmatism and higher aberrations. ${ }^{9}$ In some of the severe cases corneal scarring occurs and patients require corneal transplantation. ${ }^{10} \mathrm{KC}$ can be diagnosed during normal eye checkup even the subject has no symptoms and is unaware of the disease. ${ }^{11}$

Regarding risk factors, both genetic and environmental are present. Environmental factors act as trigger in genetically predisposed individuals. Common environmental factors involved are atopy, eye rubbing, ultraviolet (UV) exposure and contact lens use. ${ }^{12,13}$ At the molecular level, there is a lack of enzymes required for the processing of reactive oxygen species (ROS) in these environmental conditions which worsen the condition leading to vision loss. ${ }^{14}$ Eye rubbing, most common risk factor, has been shown to be associated with $\mathrm{KC}$ development in a number of studies across the globe. ${ }^{15}$ Furthermore, studies have shown that eye rubbing associated with $\mathrm{KC}$ is of long duration compared to the eye rubbing of infection or common allergy. ${ }^{16}$ Chronic eye rubbing leads to mechanical damage and history of compelling eye rubbing habit is strongly associated with $\mathrm{KC} .{ }^{17}$ The mechanical trauma generates high level of matrix mettaloproteins-1 (MMP1) and matrix metalloproteins-13 (MMP13), interlukin-6 and tumor necrosis factor alpha from epithelial and stromal cells. ${ }^{18}$ The release of these cytokines initiate process which cause appearance of many symptoms related to KC. Atopy, consisting of asthma, eczema and allergy, is a hypersensitivity reaction shown to be associated with $\mathrm{KC}$ development and progression.

A number of studies have been conducted across the globe to report the risk factors for KC. Some of the studies have found strong association for above risk factors with $\mathrm{KC}$ while some studies did not find any association so there is not a consensus in research community. The present will systematically review all the studies which have shown positive association of above risk factors and $\mathrm{KC}$. We will also do the meta-analysis to determine the effect size of the association.

\section{METHODS}

The study was a systematic review and meta-analysis of the related past studies. We used preferred reporting items for systematic reviews and meta-analyses (PRISMA) guidelines for conducting and reporting our systematic review (SR) and meta-analysis (MA).

\section{Duration and setting}

The total time in conducting SR and performing MA took 120 hours of two independent investigators. The study was conducted from 15 February 2021 to 15 March 2021.

\section{Literature search}

First, we devised a comprehensive literature search strategy. The keywords used in different combinations for search purposes were "keratoconus," "risk factors", "association", "atopic triad", "asthma", "allergy", "ecmeza" and "eye rubbing". We searched in the following electronic databases: Google scholar, Pubmed, Embase and science direct.

Two investigator independently screened abstracts and title for relevant studies. In this first round of article selection, investigators selected large number of articles only by reviewing titles and reading abstracts. For final selection of articles, we passed each article from our already prepared inclusion and exclusion criteria

\section{Inclusion criteria}

All articles showing association of our studied risk factors with KC development; articles were either case-control, cohorts, and cross-sectional studies; the language of articles published was English; and there was availability of quantitative data to determine risk factors association were included.

\section{Exclusion criteria}

Single case reports; articles in languages other than English language; and articles did not provide any quantitative data as measure of risk factors association were excluded.

\section{Data extraction}

Once all articles have been finalized, two investigators independently reviewed full texts of articles and extracted the relevant information. The data extracted was study characteristics such as ID, date of publication, authors' information, country where study was conducted and type of study. Patient related data extracted was: number of patients, mean age, gender distribution, and race if mentioned. The data extracted for conduction metaanalysis was odds ratio (OR) and their 95\% confidence interval (CI). If odds ratio was not mentioned directly other quantitative information about association was extracted and later OR and their 95\% CIs were calculated indirectly.

\section{Assessment of quality and grading}

The quality of articles is also very important in metaanalysis. We assessed quality of each article using The Newcastle-Ottawa scale (NOS). According to this scale, score of $>7$ was considered as high quality study, score in 
between 5-7 was medium quality and $<5$ score was considered low quality study. Two investigators separately assessed the quality of each article. Any discrepancy was resolved with discussion.

\section{Statistical analysis}

The statistical analysis performed was meta-analysis using forest plots. We used OR and their respective 95\% CIs of each study to draw forest plot. Furthermore, we also determined the heterogeneity in studies using $\mathrm{X}^{2}$ and $\mathrm{I}^{2}$ tests. The value of $X^{2}>50 \%$ was considered as the presence of heterogeneity. In such case random effect model was applied to determine the final effect size. Finally, publication was determined using funnel plot analysis. All the statistical analysis was performed in Rev Man software.

\section{RESULTS}

The online databases literature search from keywords resulted in 1690 articles. Further screening based on titles and abstracts reading excluded 1430 articles and yielded 260 articles. After reviewing full text articles and applying exclusion and inclusion criteria we were left with 35 articles which were included in qualitative synthesis in the form of tables and quantitative synthesis in the form of meta-analysis. ${ }^{19-53}$ The steps of search results and literature selection are summarized in a PRISMA diagram (Figure $1)$.

\section{Study and patient characteristics}

Our study included 35 studies related to risk factors association with KC. Most of the studies (22) were about atopic triad as risk factor for $\mathrm{KC}$ while the remaining 13 were related to eye rubbing as risk factor for $\mathrm{KC}$. The majority of atopic triad related studies were about allergy (9 studies). 7 of them were about asthma and the remaining 6 were eczema as risk factor for KC. Furthermore, studies had diverse designs; 21 were case control, 9 were cross sectional, and 5 had retrospective study design.
Regarding the country where studies were conducted, we found 7 studies were conducted in the US, 5 in India, 5 in Japan, 4 in Middle East, and 2 each in China, Brazil, Indonesia, Hongkong, France, and Italy. The number of subjects in all studies combined was 1786 . Out of these, 872 were eye rubbing subjects, 372 allergic rhinitis, 223 eczema, and the remaining 319 subjects were related to asthma.

There were 13 studies related to eye rubbing as risk factor. The total number of patients 932, among which $42 \%$ were women, and $58 \%$ were men. Most of the patients were Asians. The mean age of the patients was 36.29 years. Table 1 summarizes the study and patient-related information.

There were 6 studies related to eczema as risk factor and total numbers of patients were 672 . Of these, $61 \%$ were men, and $39 \%$ were women. The mean age of the patients was 32.31 years. The characteristics of these studies and the patients are summarized in Table 2. There were 7 studies related to asthma as risk facts. The total number of patients was 632. The mean age of the patients was 29.36 years. There were 9 studies related to allergy as risk factor for $\mathrm{KC}$ and 1396 patients. Of these, $37 \%$ were women and $63 \%$ men. The mean age was 36.21 years. The characteristics of the patients and these studies are summarized in Table 2.

\section{Meta-analysis}

First we performed the meta-analysis of all the risk factors combined and the results in the form of forest plot are shown in Figure 2. From all the studies, 31 were statistically significant for $\mathrm{KC}$ group and 14 were statistically significant for non $\mathrm{KC}$ ground but the overall effect size was found to be OR 2.20 with a $95 \%$ CI of $1.84-$ 2.64 with the $\mathrm{p}$ value of 0.0001 .

The overall effect size was significant for the $\mathrm{KC}$ group, implying that the studied risk factors have positive association with the development of $\mathrm{KC}(\mathrm{p}=0.0001)$.

Table 1: Characteristics of eye rubbing studies and patients.

\begin{tabular}{|c|c|c|c|c|c|c|}
\hline $\begin{array}{l}\text { First } \\
\text { authors }\end{array}$ & $\begin{array}{l}\text { Publication } \\
\text { year }\end{array}$ & $\begin{array}{l}\text { Sample } \\
\text { size }\end{array}$ & Study design & Condition & $\begin{array}{l}\text { Patient characteristics (age } \\
\text { in years and female: male) }\end{array}$ & OR $(95 \% \mathrm{CI})$ \\
\hline Kaya & 2007 & 120 & Case control & Eye rubbing & 32.7, M $26(53.1 \%)$ & $1.99(2.11,4.32)$ \\
\hline Naderan & 2015 & 322 & Case control & Eye rubbing & $29.36 \pm 6.32$, F $45.5:$ M 55.5 & $5.38(2.06,14.05)$ \\
\hline Shenor & 2014 & 244 & $\begin{array}{l}\text { Cross } \\
\text { sectional }\end{array}$ & Eye rubbing & $25.65 \pm 6.32$, F 67: M 33 & $3.09(2.17,4.00)$ \\
\hline Gordan & 2013 & 365 & Case control & Eye rubbing & $18.98 \pm 5.55$, F $63:$ M 33 & $2.31(4.25,25.04)$ \\
\hline Milodot & 2011 & 981 & $\begin{array}{l}\text { Cross } \\
\text { sectional }\end{array}$ & Eye rubbing & $23.15 \pm 3.22$, F $55:$ M 45 & $2.15(0.59,7.82)$ \\
\hline Gordan & 2015 & 217 & Case control & Eye rubbing & $33.31 \pm 6.95$, F 12.63: M 87.37 & $1.96(1.68,6.77)$ \\
\hline Hashemi & 2014 & 200 & $\begin{array}{l}\text { Cross } \\
\text { sectional }\end{array}$ & Eye rubbing & $31.21 \pm 12.56$, F $65:$ M 35 & $3.35(2.35,4.77)$ \\
\hline Virgilo & 2017 & 93 & Case control & Eye rubbing & $32.8 \pm 69.6$, F $43:$ M 57 & $1.33(1.60,24.30)$ \\
\hline Otavia & 2020 & 58 & Case control & Eye rubbing & $23.54 \pm 2.32$, F $32.65:$ M 67.35 & $3.35(2.35,4.77)$ \\
\hline
\end{tabular}




\begin{tabular}{|c|c|c|c|c|c|c|}
\hline $\begin{array}{l}\text { First } \\
\text { authors }\end{array}$ & $\begin{array}{l}\text { Publication } \\
\text { year }\end{array}$ & $\begin{array}{l}\text { Sample } \\
\text { size }\end{array}$ & Study design & Condition & $\begin{array}{l}\text { Patient characteristics (age } \\
\text { in years and female: male) }\end{array}$ & OR $(95 \% \mathrm{CI})$ \\
\hline Zaki & 2016 & 44 & ctive & Eye rubbing & 36.54ะ9.65, F 32: M 68 & $2.52(5.68,18.69)$ \\
\hline Naderan & 2017 & 922 & Case control & Eye rubbing & $24.55 \pm 6.23$, F $37.12:$ M 62.88 & $1.98(0.13,3.96)$ \\
\hline Jafri & 2004 & 87 & Case series & Eye rubbing & $25.56 \pm 6.32$, F $66.5:$ M 33.5 & $2.36(2.99,11.65)$ \\
\hline Moon & 2020 & 9322 & Cohort & Eye rubbing & $33.69 \pm 5.68$, F $12.6:$ M 87.4 & $1.72(1.36,7.99)$ \\
\hline
\end{tabular}

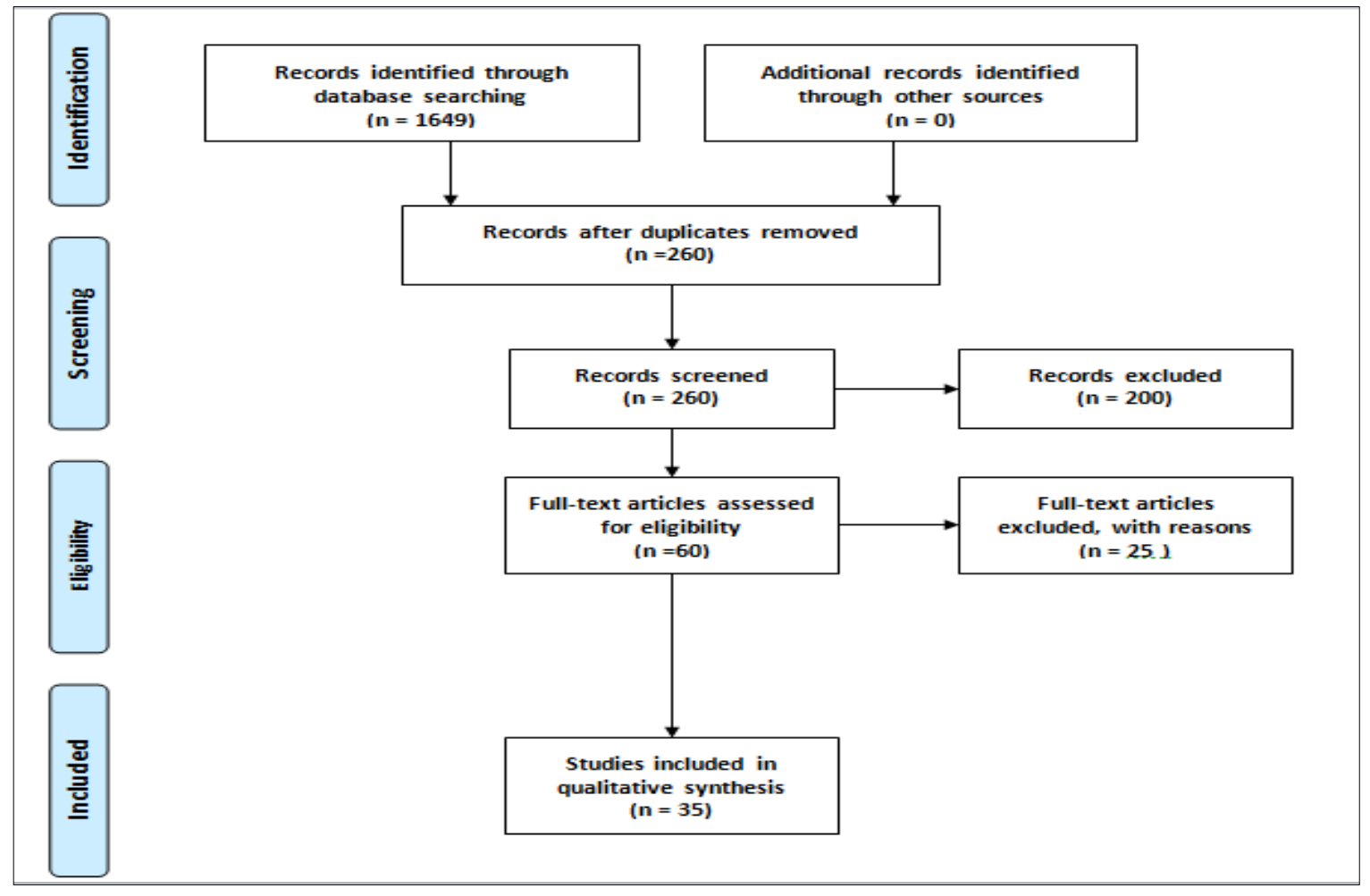

Figure 1: Summary of the literature search process.

Table 2: Characteristics of atopic triad studies and patients.

\begin{tabular}{|c|c|c|c|c|c|c|}
\hline $\begin{array}{l}\text { First } \\
\text { authors }\end{array}$ & $\begin{array}{l}\text { Publication } \\
\text { year }\end{array}$ & $\begin{array}{l}\text { Sample } \\
\text { size }\end{array}$ & Study design & Condition & $\begin{array}{l}\text { Patient characteristics } \\
\text { (age in years and } \\
\text { female: male) }\end{array}$ & OR $(95 \% \mathrm{CI})$ \\
\hline Zaki & 2008 & 44 & Retrospective & Eczema & 22.12, F 72.31: M 27.69 & $3.33(3.45,12.55)$ \\
\hline Charles & 2005 & 65 & Cross sectional & Eczema & $\begin{array}{l}35.69 \pm 9.6, \text { F } 32.69: \mathrm{M} \\
67.31\end{array}$ & $1.44(1.21,6.95)$ \\
\hline Owens & 2003 & 87 & Cross sectional & Eczema & $\begin{array}{l}29.98 \pm 6.65, \text { F } 65.5: M \\
34.5\end{array}$ & $0.89(2.33,1.52)$ \\
\hline Gordan & 2015 & 45 & Cross sectional & Eczema & 28.39, F 55.99: M 44.11 & $3.21(1.11,6.99)$ \\
\hline Naderan & 2017 & 922 & 922 & Eczema & $\begin{array}{l}30.31 \pm 6.33, \text { F } 12.8: M \\
88.2\end{array}$ & $2.22(4.55,16.99)$ \\
\hline Naderan & 2015 & & Case control & Eczema & $\begin{array}{l}23.15 \pm 4.36, \text { F } 63.98: M \\
27.2\end{array}$ & $2.33(1.21,6.56)$ \\
\hline Namet & 2010 & 400 & Case control & Asthma & $\begin{array}{l}18.69 \pm 6.32, \text { F 33.66: M } \\
66.44\end{array}$ & $3.33(1.29,6.29)$ \\
\hline Woodward & 2015 & 66 & Case control & Asthma & $\begin{array}{l}21.69 \pm 12.3 \text {, F 33.6: M } \\
67.3\end{array}$ & $1.36(2.33,7.59)$ \\
\hline Srujana & 2011 & 415 & Case control & Asthma & $33.31 \pm 6.98$, F $88:$ M 12 & $1.89(3.68,11.56)$ \\
\hline Waked & 2012 & 1566 & Cross sectional & Asthma & $19.89 \pm 6.33$, F $63:$ M 37 & $3.21(1.99,5.32)$ \\
\hline Namet & 2010 & 88 & Cross sectional & Asthma & $33.68 \pm 6.32$, F $71:$ M 29 & $2.33(0.12,4.33)$ \\
\hline Naderan & 2015 & 215 & Case control & Asthma & $\begin{array}{l}31.22 \pm 6.32, \text { F } 63.98: M \\
36.02\end{array}$ & $3.11(1.11 .6 .39)$ \\
\hline
\end{tabular}




\begin{tabular}{|c|c|c|c|c|c|c|}
\hline $\begin{array}{l}\text { First } \\
\text { authors }\end{array}$ & $\begin{array}{l}\text { Publication } \\
\text { year }\end{array}$ & $\begin{array}{l}\text { Sample } \\
\text { size }\end{array}$ & Study design & Condition & $\begin{array}{l}\text { Patient characteristics } \\
\text { (age in years and } \\
\text { female: male) }\end{array}$ & OR $(95 \% \mathrm{CI})$ \\
\hline Francis & 1989 & 512 & Case control & Asthma & 26.65, F 48.9: M 51.1 & $0.58(3.2,9.89)$ \\
\hline Gordan & 2013 & 230 & Case control & Allergy & $\begin{array}{l}32.69 \pm 6.39, \text { F 53.69: M } \\
46.31\end{array}$ & $3.29(1.32,6.33)$ \\
\hline Namet & 1989 & 188 & Case control & Allergy & 21.33ะ3.21, F 64: M 36 & $2.33(0.16,4.15)$ \\
\hline Naderan & 2015 & 98 & Case control & Allergy & $\begin{array}{l}35.69 \pm 9.68, \text { F } 56.6: \mathrm{M} \\
45.4\end{array}$ & $4.33(1.32,6.99)$ \\
\hline Shenor & 2014 & 844 & Case control & Allergy & $22.32 \pm 3.22$, F $66:$ M 44 & $1.33(0.11,3.69)$ \\
\hline Moran & 2020 & 215 & Case control & Allergy & $\begin{array}{l}34.69 \pm 9.28, \text { F } 53.99: M \\
46.11\end{array}$ & $4.32(1.22,6.88)$ \\
\hline Milodot & 2011 & 89 & Cross sectional & Allergy & $\begin{array}{l}17.69 \pm 3.65, \text { F } 22.56: M \\
27.34\end{array}$ & $1.34(2.22,9.65)$ \\
\hline Woodward & 2015 & 2036 & Cross sectional & Allergy & $\begin{array}{l}28.88 \pm 12.36, \text { F } 45.36: M \\
54.64\end{array}$ & $5.113 .65,11.11)$ \\
\hline Gordan & 2015 & 65 & Case control & Allergy & $\begin{array}{l}30.22 \pm 3.69, \text { F } 43.64: M \\
52.36\end{array}$ & $1.26(0.07,3.21)$ \\
\hline Hashemi & 2014 & 632 & Case control & Allergy & $\begin{array}{l}32.25 \pm 9.56, \text { F } 69.9: \mathrm{M} \\
31.1\end{array}$ & $2.01(1.99,7.89)$ \\
\hline
\end{tabular}

\begin{tabular}{|c|c|c|c|c|c|c|c|c|c|}
\hline Study or Subgroup & \multicolumn{2}{|c|}{ Experimental } & \multicolumn{2}{|c|}{ Control } & Weight & $\begin{array}{l}\text { Odds Ratio } \\
\text { IV, Random, } 95 \% \mathrm{Cl}\end{array}$ & \multicolumn{3}{|c|}{$\begin{array}{c}\text { Odds Ratio } \\
\text { IV, Random, } 95 \% \mathrm{Cl}\end{array}$} \\
\hline charles, 2005 eczema & 68 & 100 & 40 & 100 & $3.1 \%$ & $3.19[1.78,5.69]$ & & & \\
\hline francis, 1989 asthma & 70 & 100 & 50 & 100 & $3.1 \%$ & $2.33[1.31,4.17]$ & & 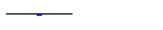 & \\
\hline Gordan,2013 allergy & 70 & 100 & 54 & 100 & $3.1 \%$ & $1.99[1.11,3.55]$ & & & \\
\hline Gordan, 2013 aye rubbing & 70 & 100 & 30 & 100 & $3.0 \%$ & $5.44[2.97,9.97]$ & & & \\
\hline gordan2015 allergy & 70 & 100 & 41 & 100 & $3.0 \%$ & $3.36[1.87,6.02]$ & & & \\
\hline gordan 2015 eczema & 70 & 100 & 35 & 100 & $3.0 \%$ & $4.33[2.39,7.84]$ & & & \\
\hline gordan 2015 eye rubbing & 70 & 100 & 63 & 100 & $3.0 \%$ & $1.37[0.76,2.47]$ & & & \\
\hline hashemi,2014 allergy & 70 & 100 & 52 & 100 & $3.1 \%$ & $2.15[1.21,3.85]$ & & & \\
\hline hashemi 2014 eye rubbing & 70 & 100 & 56 & 100 & $3.1 \%$ & $1.83[1.02,3.28]$ & & & \\
\hline jafri, 2004 eye rubbing & 70 & 100 & 65 & 100 & $3.0 \%$ & $1.26[0.69,2.27]$ & & & \\
\hline kaya, 2007 eye rubbing & 70 & 100 & 34 & 100 & $3.0 \%$ & $4.53[2.50,8.21]$ & & & \\
\hline milodot2011 allergy & 70 & 100 & 53 & 100 & $3.1 \%$ & $2.07[1.16,3.70]$ & & & \\
\hline moon eye rubbing & 70 & 100 & 39 & 100 & $3.0 \%$ & $3.65[2.03,6.56]$ & & & \\
\hline moran, 2020 allergy & 70 & 100 & 49 & 100 & $3.1 \%$ & $2.43[1.36,4.34]$ & & $=$ & \\
\hline Naderan 2015 allergy & 70 & 100 & 42 & 100 & $3.1 \%$ & $3.22[1.80,5.78]$ & & & \\
\hline Naderan 2015 asthma & 70 & 100 & 61 & 100 & $3.0 \%$ & $1.49[0.83,2.68]$ & & & \\
\hline Naderan 2015 eczema & 70 & 100 & 55 & 100 & $3.1 \%$ & $1.91[1.07,3.41]$ & & & \\
\hline Naderan 2015 eye rubbing & 70 & 100 & 73 & 100 & $2.9 \%$ & $0.86[0.47,1.60]$ & & - & \\
\hline naderan eczema & 70 & 100 & 64 & 100 & $3.0 \%$ & $1.31[0.73,2.37]$ & & & \\
\hline naderan eye rubbing & 70 & 100 & 40 & 100 & $3.0 \%$ & $3.50[1.95,6.29]$ & & & \\
\hline namet, 2010allergy & 70 & 100 & 41 & 100 & $3.0 \%$ & $3.36[1.87,6.02]$ & & & \\
\hline namet, 2010 asthma & 70 & 100 & 30 & 100 & $3.0 \%$ & $5.44[2.97,9.97]$ & & & \\
\hline otavia eye rubbing & 70 & 100 & 63 & 100 & $3.0 \%$ & $1.37[0.76,2.47]$ & & & \\
\hline owens 2003 eczema & 70 & 100 & 50 & 100 & $3.1 \%$ & $2.33[1.31,4.17]$ & & & \\
\hline shenor,2014 allergy & 70 & 100 & 42 & 100 & $3.1 \%$ & $3.22[1.80,5.78]$ & & & \\
\hline shenor, 2014 aye rubbing & 70 & 100 & 41 & 100 & $3.0 \%$ & $3.36[1.87,6.02]$ & & & \\
\hline srujana,2011 asthma & 70 & 100 & 48 & 100 & $3.1 \%$ & $2.53[1.41,4.52]$ & & & \\
\hline virgilo eye rubbing & 70 & 100 & 65 & 100 & $3.0 \%$ & $1.26[0.69,2.27]$ & & - & \\
\hline waked asthma & 70 & 100 & 52 & 100 & $3.1 \%$ & $2.15[1.21,3.85]$ & & & \\
\hline woodward, 2015 allergy & 70 & 100 & 58 & 100 & $3.1 \%$ & $1.69[0.94,3.03]$ & & & \\
\hline woodward,2015 asthma & 70 & 100 & 80 & 100 & $2.8 \%$ & $0.58[0.30,1.12]$ & & & \\
\hline zaki,2016 eczema & 70 & 100 & 50 & 100 & $3.1 \%$ & $2.33[1.31,4.17]$ & & & \\
\hline zaki,2016 eye rubbing & 70 & 100 & 76 & 100 & $2.9 \%$ & $0.74[0.39,1.38]$ & & & \\
\hline Total $(95 \% \mathrm{Cl})$ & & 3300 & & 3300 & $100.0 \%$ & $2.20[1.84,2.64]$ & & 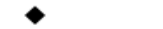 & \\
\hline \multirow{2}{*}{\multicolumn{7}{|c|}{$\begin{array}{l}\text { Total events } \\
\text { Heterogeneity: } \mathrm{Tau}^{2}=0.19 ; \mathrm{Chi}^{2}=99.06, \mathrm{df}=32(\mathrm{P}<0.00001) ; \mathrm{I}^{2}=68 \%\end{array}$}} & & & \\
\hline & & & & & & & 0.01011 & $\begin{array}{c}10 \\
\text { Favours [control] }\end{array}$ & 100 \\
\hline
\end{tabular}

Figure 2: Meta-analysis of all conditions as risk factor for $\mathrm{KC}$.

\begin{tabular}{|c|c|c|c|c|c|c|c|c|c|}
\hline \multirow[b]{2}{*}{ Study or Subgroup } & \multicolumn{2}{|c|}{ control } & \multicolumn{2}{|c|}{ experimental } & \multirow[b]{2}{*}{ Weight } & \multirow{2}{*}{$\begin{array}{c}\text { Odds Ratio } \\
\text { M-H, Fixed, } 95 \% \mathrm{Cl}\end{array}$} & \multirow{2}{*}{\multicolumn{3}{|c|}{$\begin{array}{c}\text { Odds Ratio } \\
\text { M-H, Fixed, } 95 \% \mathrm{Cl}\end{array}$}} \\
\hline & Events & Total & Events & Total & & & & & \\
\hline Gordan, 2013 aye rubbing & 70 & 100 & 30 & 100 & $5.2 \%$ & $5.44[2.97,9.97]$ & & & \\
\hline gordan 2015 eye rubbing & 70 & 100 & 63 & 100 & $10.9 \%$ & $1.37[0.76,2.47]$ & & & \\
\hline hashemi 2014 eye rubbing & 70 & 100 & 56 & 100 & $9.7 \%$ & $1.83[1.02,3.28]$ & & $\rightarrow$ & \\
\hline kaya, 2007 eye rubbing & 70 & 100 & 34 & 100 & $5.9 \%$ & $4.53[2.50,8.21]$ & & & \\
\hline moon eye rubbing & 70 & 100 & 39 & 100 & $6.7 \%$ & $3.65[2.03,6.56]$ & & & \\
\hline Naderan 2015 eye rubbing & 70 & 100 & 73 & 100 & $12.6 \%$ & $0.86[0.47,1.60]$ & & & \\
\hline naderan eye rubbing & 70 & 100 & 40 & 100 & $6.9 \%$ & $3.50[1.95,6.29]$ & & & \\
\hline otavia eye rubbing & 70 & 100 & 63 & 100 & $10.9 \%$ & $1.37[0.76,2.47]$ & & - & \\
\hline shenor, 2014 aye rubbing & 70 & 100 & 41 & 100 & $7.1 \%$ & $3.36[1.87,6.02]$ & & & \\
\hline virgilo eye rubbing & 70 & 100 & 65 & 100 & $11.2 \%$ & $1.26[0.69,2.27]$ & & - & \\
\hline zaki,2016 eye rubbing & 70 & 100 & 76 & 100 & $13.1 \%$ & $0.74[0.39,1.38]$ & & & \\
\hline Total $(95 \% \mathrm{Cl})$ & & 1100 & & 1100 & $100.0 \%$ & $2.09[1.76,2.49]$ & & $\bullet$ & \\
\hline Total events & 770 & & 580 & & & & & & \\
\hline $\begin{array}{l}\text { Heterogeneity: } \mathrm{Chi}^{2}=50.61 \text {, } \\
\text { Test for overall effect: } Z=8.2\end{array}$ & $\begin{array}{l}f=10(P \\
P(P<0.0\end{array}$ & $\begin{array}{l}<0.000 \\
0001)\end{array}$ & 001); $\left.\right|^{2}=8$ & & & & $\begin{array}{ccc} \\
0.01 & 0.1 \\
& \text { Favours [experimental] }\end{array}$ & $\begin{array}{cc}10 \\
\text { Favours [control] }\end{array}$ & 100 \\
\hline
\end{tabular}

Figure 3: Meta-analysis of eye rubbing studies as risk factor for $\mathrm{KC}$. 


\section{Eye rubbing as a risk factor for $\mathrm{KC}$}

From 13 of eye rubbing related studies, 9 were significant for $\mathrm{KC}$ group and 4 were statistically significant for non $\mathrm{KC}$ group. When analyzed in meta-analysis we found the overall effect size of 2.09 with a $95 \%$ CI of 1.76-2.49. Overall, eye rubbing was a risk factor for $\mathrm{KC}$ with a $\mathrm{p}$ value of 0.00001 (Figure 3).

\section{Atopic triad as a risk factor for $\mathrm{KC}$}

When all atopic triad (allergy, asthma, and eczema) related studies were analyzed in meta-analysis, an overall effect size of 2.34 was found with $95 \%$ CI of (2.06-2.66). 5 studies were significant for non $\mathrm{KC}$ group and the remaining were significant for $\mathrm{KC}$ group. Overall, atopic triad was a risk factor for $\mathrm{KC}$ with a $\mathrm{p}$ value of $(0.00001)$ (Figure 4).

\begin{tabular}{|c|c|c|c|c|c|c|c|c|c|}
\hline Study or Subgroup & \multicolumn{2}{|c|}{ Experimental } & \multicolumn{2}{|c|}{ Control } & Weight & \multirow{2}{*}{$\begin{array}{c}\text { Odds Ratio } \\
\text { M-H, Fixed, } 95 \% \mathrm{Cl}\end{array}$} & \multicolumn{3}{|c|}{$\begin{array}{c}\text { Odds Ratio } \\
\text { M-H, Fixed, } 95 \% \mathrm{Cl}\end{array}$} \\
\hline charles, 2005 eczema & 70 & 100 & 40 & 100 & $3.8 \%$ & & & & \\
\hline francis, 1989 asthma & 70 & 100 & 50 & 100 & $4.8 \%$ & $2.33[1.31,4.17]$ & & $\longrightarrow$ & \\
\hline Gordan,2013 allergy & 70 & 100 & 54 & 100 & $5.2 \%$ & $1.99[1.11,3.55]$ & & $\longrightarrow$ & \\
\hline gordan 2015 allergy & 70 & 100 & 41 & 100 & $3.9 \%$ & $3.36[1.87,6.02]$ & & $\longrightarrow$ & \\
\hline gordan 2015 eczema & 70 & 100 & 35 & 100 & $3.3 \%$ & $4.33[2.39,7.84]$ & & & \\
\hline hashemi, 2014 allergy & 70 & 100 & 52 & 100 & $5.0 \%$ & $2.15[1.21,3.85]$ & & $\longrightarrow$ & \\
\hline milodot 2011 allergy & 70 & 100 & 54 & 100 & $5.2 \%$ & $1.99[1.11,3.55]$ & & $\longrightarrow$ & \\
\hline moran,2020 allergy & 70 & 100 & 49 & 100 & $4.7 \%$ & $2.43[1.36,4.34]$ & & & \\
\hline Naderan 2015 allergy & 70 & 100 & 42 & 100 & $4.0 \%$ & $3.22[1.80,5.78]$ & & & \\
\hline Naderan 2015 asthma & 70 & 100 & 61 & 100 & $5.8 \%$ & $1.49[0.83,2.68]$ & & & \\
\hline Naderan 2015 eczema & 70 & 100 & 55 & 100 & $5.2 \%$ & $1.91[1.07,3.41]$ & & & \\
\hline naderan eczema & 70 & 100 & 64 & 100 & $6.1 \%$ & $1.31[0.73,2.37]$ & & - & \\
\hline namet,2010allergy & 70 & 100 & 41 & 100 & $3.9 \%$ & $3.36[1.87,6.02]$ & & & \\
\hline namet, 2010 asthma & 70 & 100 & 30 & 100 & $2.9 \%$ & $5.44[2.97,9.97]$ & & & \\
\hline owens 2003 eczema & 70 & 100 & 50 & 100 & $4.8 \%$ & $2.33[1.31,4.17]$ & & $\rightarrow$ & \\
\hline shenor,2014 allergy & 70 & 100 & 42 & 100 & $4.0 \%$ & $3.22[1.80,5.78]$ & & & \\
\hline srujana,2011 asthma & 70 & 100 & 48 & 100 & $4.6 \%$ & $2.53[1.41,4.52]$ & & - & \\
\hline waked asthma & 70 & 100 & 52 & 100 & $5.0 \%$ & $2.15[1.21,3.85]$ & & - & \\
\hline woodward,2015 allergy & 70 & 100 & 58 & 100 & $5.5 \%$ & $1.69[0.94,3.03]$ & & & \\
\hline woodward,2015 asthma & 70 & 100 & 80 & 100 & $7.6 \%$ & $0.58[0.30,1.12]$ & & & \\
\hline zaki,2016 eczema & 70 & 100 & 50 & 100 & $4.8 \%$ & $2.33[1.31,4.17]$ & & & \\
\hline Total $(95 \% \mathrm{Cl})$ & & 2100 & & 2100 & $100.0 \%$ & $2.34[2.06,2.66]$ & & $\bullet$ & \\
\hline Total events & 1470 & & 1048 & & & & & & \\
\hline \multirow{2}{*}{\multicolumn{7}{|c|}{$\begin{array}{l}\text { Heterogeneity: } \mathrm{Chi}^{2}=44.66, \mathrm{df}=20(\mathrm{P}=0.001) ; \mathrm{I}^{2}=55 \% \\
\text { Test for overall effect: } Z=13.18(P=0.00001)\end{array}$}} & 0.01 & 10 & $\overrightarrow{100}$ \\
\hline & & & & & & & Favours [experir & Favours [control] & \\
\hline
\end{tabular}

Figure 4: Meta-analysis of atopic triad conditions as risk factor for $\mathrm{KC}$.

\section{DISCUSSION}

The present study is a meta-analysis to determine how strongly risk factors eye rubbing and atopic triad are associated with the development of KC. This is the first systematic review and meta-analysis on the topic and we were able to identify 35 studies to be included in the metaanalysis. The numbers of articles were enough with reasonable number of patients for a good meta-analysis.

$\mathrm{KC}$, a corneal disease, has been found to be associated with a number of genetic and environmental factors. Eye rubbing is considered as a strongest risk factor for KC. Our analysis has found strong association of eye rubbing with the development of $\mathrm{KC}$ with $\mathrm{p}$ value of 0.0001 and overall combined OR of 2.09. Our results are similar to the number of other studies. For example Bawazeer had found strong association with OR of $5.38 .{ }^{48}$ Similarly, 2 more important studies had also found strong association with OR of 10.31 and 6.30. ${ }^{49,50}$ There were also some studies who did not find any association for eye rubbing as risk factor for KC. In our analysis, we found 4 studies showing non association of eye rubbing as risk factor for $\mathrm{KC}$. There can be a number of reasons for the discrepancy. One of the most important reasons is the criteria for abnormal eye rubbing..$^{51}$
Atopic triad (AT), a combination of allergy, eczema, and asthma, is another important risk factor for KC. Our analysis found positive association for AT with $\mathrm{KC}$ development and progression with $\mathrm{p}$ value of 0.0001 and overall effect size of OR 2.34. These results are similar to other studies with OR ranging from 1.59 to $6.330 .^{52,53}$ Other studies did not find any association for AT as risk factor of KC. Atopy is another condition in which individuals have genetic tendency to develop allergy, asthma and eczema. There are controversial results regarding atopy and its association with KC. Some of the studies have shown strong association while other do not consider atopy as risk factor for KC. The reasons for varied results is a disagreement on the definition of atopy in literature. Some studies consider all three conditions, asthma, eczema and allergy in atopy while some consider only asthma or only allergy. ${ }^{53,54}$

Like most of other studies our studies also have some limitations. First, there is a significant amount of cross sectional studies involved for conducting meta-analysis. The evidence presented by cross-sectional studies is of poorer quality compared to that presented by case-control studies. Besides, the studies were heterogeneous. Although the studies considered here had a number of confounding variables such as gender, age, and ethnicity, we did not perform a subgroup analysis based on these variables, which can affect the results. Finally, the studies had a publication bias as well. 


\section{CONCLUSION}

Based on this meta-analysis it can be concluded that eye rubbing, and atopic triad (allergic rhinitis, asthma, and eczema) are important risk factors for KC. Furthermore, male gender is more affected with the condition compared to the females. There is a need for more high quality controlled studies to determine association.

\section{Funding: No funding sources}

Conflict of interest: None declared

Ethical approval: Not required

\section{REFERENCES}

1. Kennedy RH, Bourne WM, Dyer JA. A 48-year clinical and epidemiologic study of keratoconus. Am J Ophthalmol. 1986;101(3):267-73.

2. Rabinowitz YS. Keratoconus. Surv Ophthalmol. 1998;42(4):297-319.

3. Jonas JB, Nangia V, Matin A, Kulkarni M, Bhojwani $\mathrm{K}$. Prevalence and associations of keratoconus in rural Maharashtra in central India: the central India eye and medical study. Am J Ophthalmol. 2009;148(5):760-5.

4. Gordon-Shaag A, Millodot M, Essa M, Garth J, Ghara M, Shneor E. Is consanguinity a risk factor for keratoconus? Optom Vis Sci. 2013;90(5):448-54.

5. Cozma I, Atherley C, James N. Influence of ethnic origin on the incidence of keratoconus and associated atopic disease in Asian and white patients. Eye (London, Engl). 2005;19(8):924-5.

6. Hashemi H, Khabazkhoob M, Yazdani N. The prevalence of keratoconus in a young population in Mashhad, Iran. Ophthalmic Physiol Opt. 2014;34(5):519-27.

7. Chang HY, Chodosh J. The genetics of keratoconus. Semin Ophthalmol. 2013;28(5-6):275-80.

8. Georgiou T, Funnell CL, Cassels-Brown A, O'Conor $\mathrm{R}$. Influence of ethnic origin on the incidence of keratoconus and associated atopic disease in Asians and white patients. Eye (London, Engl). 2004;18(4):379-83.

9. Cozma I, Atherley C, James N. Influence of ethnic origin on the incidence of keratoconus and associated atopic disease in Asian and white patients. Eye (London, Engl). 2005;19(8):924-5.

10. Pramanik S, Musch DC, Sutphin JE, Farjo AA. Extended long-term outcomes of penetrating keratoplasty for keratoconus. Ophthalmology. 2006;113(9):1633-8.

11. Millodot M, Shneor E, Albou S, Atlani E, GordonShaag A. Prevalence and associated factors of keratoconus in Jerusalem: a cross-sectional study. Ophthalmic Epidemiol. 2011;18(2):91-7.

12. Davidson AE, Hayes S, Hardcastle AJ, Tuft SJ. The pathogenesis of keratoconus. Eye (Lond). 2014;28(2):189-95.
13. Macsai MS, Varley GA, Krachmer JH. Development of keratoconus after contact lens wear. Patient characteristics. Arch Ophthalmol. 1990;108:534-8.

14. Gondhowiardjo TD, van Haeringen NJ, VölkerDieben HJ, Beekhuis HW, Kok JH, van Rij G, Pels L, Kijlstra A. Analysis of corneal aldehyde dehydrogenase patterns in pathologic corneas. Cornea. 1993;12(2):146-54.

15. Gordon-Shaag A, Shneor E, Millodot M. The epidemiology and etiology of Keratoconus. Int $\mathbf{J}$ Keratoconus Ectatic Corneal Dis. 2012;1(1):7-15.

16. McMonnies $\mathrm{CW}$, Boneham GC. Keratoconus, allergy, itch, eye-rubbing and hand-dominance. Clin Exp Optom. 2003;86(6):376-84.

17. Mc Monnies CW. Abnormal rubbing and keratectasia. Eye Contact Lens. 2007;33(6):265-71.

18. Sharma N, Rao K, Maharana PK. Ocular allergy and keratoconus. Indian J Ophthalmol. 2013;61:407-9.

19. Georgiou T, Funnell CL, Cassels-Brown A. Influence of ethnic origin on the incidence of keratoconus and associated atopic disease in Asians and white patients. Eye (Lond). 2004;18:379-83.

20. Bawazeer AM, Hodge WG, Lorimer B. Atopy and keratoconus: a multivariate analysis. $\mathrm{Br} \quad \mathrm{J}$ Ophthalmol. 2000;84:834-6.

21. McMonnies CW. Abnormal rubbing and keratectasia. Eye Contact Lens. 2007;33:265-71.

22. Macsai MS, Varley GA, Krachmer JH. Development of keratoconus after contact lens wear. Patient characteristics. Arch Ophthalmol. 1990;108:534-8.

23. Abu-Amero KK, Kalantan H, Al-Muammar AM. Analysis of the VSX1 gene in keratoconus patients from Saudi Arabia. Mol Vis. 2011;17:667-72.

24. Kaya V, Utine CA, Altunsoy M. Evaluation of corneal topography with Orbscan II in first-degree relatives of patients with keratoconus. Cornea. 2008;27:531-4.

25. Reeves SW, Ellwein LB, Kim T. Keratoconus in the medicare population. Cornea. 2009;28:40-2.

26. Waked N, Fayad A, Fadlallah A. Keratoconus screening in a Lebanese students' population [in French]. J Fr Ophtalmol. 2012;35:23-9.

27. Altman A, Lorimer B, Hodge W. Atopy and other risk factors for keratoconus. Invest Ophthalmol Vis Sci. 1997;38.

28. Gordon-Shaag A, Millodot M, Essa M. Is consanguinity a risk factor for keratoconus? Optom Vis Sci. 2013;90:448-54.

29. Kaiserman PY, Assouline M, Ducoussau F. Prevalence of keratoconus. Vis Res. 1995;35:2311.

30. Merdler I, Hassidim A, Sorkin N. Keratoconus and allergic diseases among Israeli adolescents between 2005 and 2013. Cornea. 2015;34:525-9.

31. Hashemi H, Khabazkhoob M, Yazdani N. The prevalence of keratoconus in a young population in Mashhad, Iran. Ophthalmic Physiol Opt. 2014;34:519-27.

32. Althomali TA, Al-Qurashi IM, Al-Thagafi SM. Prevalence of keratoconus among patients seeking 
laser vision correction in Taif area of Saudi Arabia. Saudi J Ophthalmol. 2018;32:114-8.

33. Jonas JB, Nangia V, Matin A. Prevalence and associations of keratoconus in rural Maharashtra in central India: the central India eye and medical study. Am J Ophthalmol. 2009;148:760-5.

34. Kosker M, Suri K, Hammersmith KM. Another look at the association between diabetes and keratoconus. Cornea. 2014;33:774-9.

35. Hofstetter HW. A keratoscopic survey of 13,395 eyes. Am J Optom Arch Am Acad Optom. 1959;36:3-11.

36. Naderan M, Naderan M, Rezagholizadeh F, Zolfaghari M, Pahlevani R, Rajabi MT. Association between diabetes and keratoconus: a case-control study. Cornea. 2014;33:1271-3.

37. Naderan M, Rajabi MT, Zarrinbakhsh P. Effect of allergic diseases on keratoconus severity. Ocul Immunol Inflamm. 2017;25:418-23.

38. Naderan M, Shoar S, Rezagholizadeh F. Characteristics and associations of keratoconus patients. Cont Lens Anterior Eye. 2015;38:199-205.

39. Shehadeh MM, Diakonis VF, Jalil SA. Prevalence of keratoconus among a palestinian tertiary student population. Open Ophthalmol J. 2015;9:172-6.

40. Gordon-Shaag A, Millodot M, Kaiserman I. Risk factors for keratoconus in Israel: a case-control study. Ophthalmic Physiol Opt. 2015;35:673-81.

41. Woodward MA, Blachley TS, Stein JD. The association between sociodemographic factors, common systemic diseases, and keratoconus: an analysis of a Nationwide heath care claims database. Ophthalmology. 2016;123:457-65.

42. Xu L, Wang YX, Guo Y. Prevalence and associations of steep cornea/keratoconus in Greater Beijing. The Beijing Eye Study. PLoS One. 2012;7;e39313.

43. Hwang S, Lim DH, Chung TY. Prevalence and incidence of keratoconus in South Korea: a Nationwide Population-based Study. Am J Ophthalmol. 2018;192:56-64.

44. Hashemi H, Beiranvand A, Khabazkhoob M. Prevalence of keratoconus in a population-based study in Shahroud. Cornea. 2013;32:1441-5.

45. Hashemi H, Khabazkhoob M, Fotouhi A. Topographic keratoconus is not rare in an Iranian population: the Tehran Eye Study. Ophthalmic Epidemiol. 2013;20:385-91.

46. Mukhtar S, Ambati BK. Pediatric keratoconus: a review of the literature. Int Ophthalmol. 2018;38:2257-66.

47. Tachibana M, Adachi W, Kinoshita S. Androgendependent hereditary mouse keratoconus: linkage to an MHC region. Invest Ophthalmol Vis Sci. 2002;43:51-7.

48. Korb DR, Leahy CD, Greiner JV. Prevalence and characteristics of eye rubbing for keratoconic and non-keratoconic subjects. Invest Ophthalmol Vis Sci. 1991;32:884.

49. Wisse RP, Kuiper JJ, Gans R. Cytokine expression in keratoconus and its corneal microenvironment: a systematic review. Ocul Surf. 2015;13:272-83.

50. Gordon-Shaag A, Millodot M, Shneor E. The epidemiology and etiology of keratoconus. Epidemiology. 2012;7-15. 20. Moher D, Liberati A, Tetzlaff $\mathbf{J}$, et al. Preferred reporting items for systematic reviews and meta-analyses: the PRISMA statement. PLoS Med. 2009;6:e1000097.

51. Ihalainen A. Clinical and epidemiological features of keratoconus genetic and external factors in the pathogenesis of the disease. Acta Ophthalmol Suppl. 1986; 178:1-64.

52. Kok YO, Tan GF, Loon SC. Review: keratoconus in Asia. Cornea. 2012;31:581-93.

53. Millodot M, Shneor E, Albou S. Prevalence and associated factors of keratoconus in Jerusalem: a cross-sectional study. Ophthalmic Epidemiol. 2011;18:91-7.

54. Chwa M, Atilano SR, Reddy V. Increased stressinduced generation of reactive oxygen species and apoptosis in human keratoconus fibroblasts. Invest Ophthalmol Vis Sci. 2006;47:1902-10.

Cite this article as: Albalawi A, Alharbi A, Alhasani H, Alharbi A, Abdullah R, Alamrani A, et al. Atopic triad (allergic rhinitis, eczema, and asthma) and eye rubbing as risk factors for keratoconus: a systematic review and meta-analysis. Int J Community Med Public Health 2021;8:2523-30. 